\title{
NEW RESULTS ON THE GEOMETRY OF TRANSLATION SURFACES
}

\author{
MARIAN IOAN MUNTEANU and ANA IRINA NISTOR
}

Department of Mathematics, University Al. I Cuza of Iaşi, Bd. Carol I, n. 11, Iaşi 700506, Romania

\begin{abstract}
In this paper we study the second mean curvature for different hypersurfaces in space forms. We furnish some examples and we remind some connections between $I I$-minimality and biharmonicity. The main result consists in proving that there are no $I I$-minimal translation surfaces in the Euclidean 3-space.
\end{abstract}

\section{Introduction}

The study of the second fundamental form $I I$ was initiated through the early papers of J. Weingarten [13], G. Darboux [3] and E. Cartan [2] where appeared for the first time notions like connection or curvature associated to $I I$. Later on, P. Erard [5] introduced the second fundamental form as metric on the surface. This is possible only when $I I$ is non-degenerate and hence it can be regarded as a (pseudo)Riemannian metric on the surface. At this point one can consider a connected smooth surface $M$ endowed with $I I$ as metric in order to study new characteristics associated to $(M, I I)$. In the classical case when the metric on the surface is given by the first fundamental form $I$, i.e. for $(M, I)$, there are well known formulae to compute the Gaussian curvature $K$ and the mean curvature $H$ in order to analyze the properties of $M$ that arise from this "measures". In a similar manner, the second Gaussian curvature denoted $K_{I I}$ and the second mean curvature, denoted $H_{I I}$, were introduced. In [2], $K_{I I}$ was introduced for the first time by E. Cartan, as the analogous of the Gaussian curvature. Concerning $H_{I I}$, it was defined by E. Glässner in [6]. An overview over the literature dedicated to the second fundamental form and the associated curvatures for different type of submanifolds in different ambient spaces can be found in [12] and its references. Regarding the 
second mean curvature, the critical points of the area functional of the second fundamental form are those surfaces for which the mean curvature of the second fundamental form vanishes. A non-developable surface is said to be II-flat if $K_{I I}=0$ and respectively II-minimal if $H_{I I}=0$. Consequently, the well known result that there are no compact minimal submanifolds in Euclidean space was discussed also in the case of the second mean curvature and in [6] it is proved that do not exist compact II-minimal surfaces in Euclidean space. Despite this non-existence result confirmed also in some other particular ambient spaces, in [12] it is proved that compact II-minimal surfaces may exist in some general ambient spaces.

In the present paper we are interested in the study of the second mean curvature for different examples of submanifolds. More exactly we study the $I I$-minimality property, equivalently, the condition $H_{I I}=0$. The classical examples in the theory of harmonic and biharmonic maps are proved to be also interesting examples concerning the $I I$-minimality property. Let us remind the following situations of classical biharmonic maps which are also $I I$-minimal, namely the standard embedding $\mathbb{S}^{n}\left(\frac{1}{\sqrt{2}}\right) \subset \mathbb{S}^{n+1}$ and the hypersurface $\mathbb{S}^{k}\left(\frac{1}{\sqrt{2}}\right) \times \mathbb{S}^{n-k}\left(\frac{1}{\sqrt{2}}\right) \subset \mathbb{S}^{n+1}$ for $k=\overline{1, n-1}$ (see [12]). In the sequel we formulate some generalizations of these results.

Returning to the theory of surfaces, in [8] it is proved that a ruled surface with nowhere vanishing Gaussian curvature is II-minimal if and only if is a piece of helicoid. A study on II-minimal affine translation surfaces written as a sum of two curves is contained in [9]. Here it is stated that there are no affine II-minimal translation surfaces of this type. As a particular case it is suggested that one can retrieve the same non-existence result concerning $I I$-minimal translation surfaces in Euclidean 3-space. The aim of this article is to give a proof of this result in the case when the two curves are situated in orthogonal planes. This result is contained in Section 3, after we acquaint the reader with the basic notions about translation surfaces and $I I$-minimality in the Preliminaries of this article. Moreover, we provide also interesting examples of $I I$-minimal hypersurfaces in different space forms.

\section{Preliminaries}

In the general theory of surfaces and hypersurfaces ordinary denoted $M$, isometrically immersed in some ambient space $(\widetilde{M}, \tilde{g})$, one can associate different "measures". Naturally, a way of describe a metric $g$ on $M$ is taking the restriction to $M$ of the matric $\tilde{g}$ from the ambient space.

Thinking now the immersion $(M, g) \hookrightarrow(\widetilde{M}, \tilde{g})$ where $M$ is a hypersurface, i.e.has codimension 1, we have the Gauss and Weingarten formulas

$$
\begin{aligned}
& \widetilde{\nabla}_{X} Y=\nabla_{X} Y+I I(X, Y) N \\
& \widetilde{\nabla}_{X} N=-A X
\end{aligned}
$$


for every $X, Y$ tangent to $M$. The corresponding Levi-Civita connections on the ambient space and on the surface are denoted by $\widetilde{\nabla}$ and $\nabla$, respectively. Moreover $I I$ is a symmetric $(1,2)$-tensor field called the second fundamental form of the surface $M$ and $A$ is a symmetric $(1,1)$-tensor field denoting the shape operator associated to the unit normal to the surface $N$. The following relation holds $I I(X, Y)=g(X, A Y)$, where $X, Y$ are vector fields tangents to $M$.

Concerning the curvature tensor $R$ on the surface and using the previous notations, recall that we use the following sign convention $R(X, Y)=\left[\nabla_{X}, \nabla_{Y}\right]-\nabla_{[X, Y]}$ for any $X, Y \in T(M)$.

Having now some basic working tools on $M$ we can construct its intrinsic and extrinsic geometry by means of the characterization of the curvatures. The most used metrics on a surface is given by the first fundamental form $I$ associated to the immersion which gives the parametrization. But, one can think of rebuilding all the corresponding geometry to $I$ by taking the second fundamental form as a new metric on the surface. One elementary condition that $I I$ must satisfy consists of non-degeneracy, namely the surface must be non-developable.

\subsection{Translation surfaces}

Let us consider a surface having the Cartesian parametrization given by

$$
\left(\begin{array}{c}
x \\
y
\end{array}\right) \mapsto A(x)\left(\begin{array}{l}
\mathbf{f}(y) \\
\mathbf{g}(y) \\
\mathbf{h}(y)
\end{array}\right)+\left(\begin{array}{c}
a(x) \\
b(x) \\
c(x)
\end{array}\right)
$$

where $A(x) \in S O(3)$ (sometimes in $O(3)$ ). This surface represents a union of "equal" curves i.e. it is the image of one curve, called generatrix, obtained by isometries of the space. Some authors call this kind of surface a surface of Darboux. Some known examples are to be mentioned, namely

1. $A=I_{3}$ : translation surfaces

2. $A=$ matrix of rotation (axe and angle are fixed), $a=b=c=0$ : rotation surfaces

3. $A$ = matrix of rotation (axe $\bar{n}$ and angle are fixed), $(a, b, c)=x \bar{n}$ : helicoidal surfaces

If the generatrix is

a. a straight line : ruled surfaces

b. a circle : circled surfaces including e.g. tubes. For a smooth curve $\gamma$, the tube of radius 1 around it is given by

$$
r(s, t)=\gamma(t)+\cos s N(t)+\sin s B(t)
$$


where $s$ is the arclength parameter, $N(s)$ and $B(s)$ are respectively the normal and the binormal of the curve. As a Darboux surface, a tube can be written as

$$
r(s, t)=\gamma(t)+A(t) \mathbb{S}^{1}
$$

where by $\mathbb{S}^{1}$ we mean the unit circle.

The special Euclidean group of the $n$-dimensional space or the Euclidean motion group $S E(n)$ is the semi-direct product of $\mathbb{R}^{n}$ with the special orthogonal group $S O(n)$. In the 3-dimensional case $S E(3)=\mathbb{R}^{3} \rtimes S O(3)$. To be more precise, for two elements $g=(a, A)$ and $h=(b, B)$ in $S E(3)$, the group multiplication and the inverse are given by $g \circ h=(a+A b, A B)$, respectively $g^{-1}=\left(-A^{T} a, A^{T}\right)$. It is also possible to represent any element of $S E(n)$ as an $(n+1) \times(n+1)$ homogeneous transformation matrix of the form $\left(\begin{array}{ll}A & a \\ O & 1\end{array}\right)$ (see e.g. [11], p.45).

A Darboux surface can be thought as the action of the 1-parameter family of $S E(3)$ to a given curve.

As we have seen, a translation surface is a "sum" of two curves. If the two curves are situated in orthogonal planes the surface can be represented as

$$
(x, y) \longmapsto(x, y, f(x)+g(y)) .
$$

Examples: planes, cylinders, hyperbolic and elliptic paraboloids, the egg box surface, Scherk surface (the only minimal translation surface in $\mathbb{E}^{3}$ ).

\subsection{II-minimality}

We dedicate this section to some very nice examples of II-minimal hypersurfaces immersed in the sphere $\mathbb{S}^{n}$ and we give a nonexistence result in the case of IIminimal hypersurfaces in the hyperbolic space $\mathbb{H}^{n}$. The motivation comes from another interesting property of these immersions intensively studied in recent years, namely the biharmonicity. Similar to the variational characterization of the mean curvature $H$, the curvature of the second fundamental form, denoted by $H_{I I}$ is introduced as a measure for the rate of change of the II-area under a normal deformation. Let us denote $M$ a $m$-dimensional hypersurface in a semi-Riemannian manifold $(\widetilde{M}, \tilde{g})$ with the second fundamental form as semi-Riemannian metric. Accordingly to [12], the mean curvature associated to the second mean curvature, $H_{I I}$ is given by

$$
H_{I I}=\frac{1}{2}\left(m H-\sum_{i=1}^{m} \tilde{g}\left(\tilde{R}\left(V_{i}, N\right) V_{i}, N\right) \kappa_{i}+\frac{\alpha}{2} \Delta_{I I} \log |\operatorname{det} A|-\alpha \operatorname{div}_{I I} \mathcal{Z}\right) .
$$

Let us explain in few words the notations used above. The classical mean curvature of the first fundamental form is denoted by $H$, the unit normal is called $N$ and by $A$ we mean the shape operator of $M$. Moreover, $V_{i}, i=\overline{1, m}$ is an orthonormal basis 
on $M$ with respect to $I I$ and by definition $\kappa_{i}=I I\left(V_{i}, V_{i}\right)= \pm 1, i=\overline{1, m}$. The vector field $\mathcal{Z}$ in $T(M)$ is defined as $\mathcal{Z}=\operatorname{tr}_{I I} B=\sum_{i=1}^{m} B\left(V_{i}, V_{i}\right) \kappa_{i}$, where the tensor $B: T(M) \times T(M) \rightarrow T(M)$ is defined as $(V, W) \mapsto A^{-1}\left\{(\widetilde{R}(V, N) W)^{\mathrm{t}}\right\}$. Here, by ${ }^{t}$ we denoted the tangential component of the corresponding vector field. The shape operator is thought here as a field of endomorphisms of each tangent spaces in points of $M$, namely $A: T(M) \rightarrow T(M), V \mapsto-\widetilde{\nabla}_{V} N$.

If the ambient space is a space form (its sectional curvature is constant), then the tensor $B$ vanishes and hence $\mathcal{Z}=0$. Moreover, if in addition the shape operator has constant determinant (and this often happens) the second mean curvature can be computed by an easier formula, namely

$$
H_{I I}=\frac{1}{2}\left(m H-\sum_{i=1}^{m} \widetilde{g}\left(\widetilde{R}\left(V_{i}, N\right) V_{i}, N\right) \kappa_{i}\right) .
$$

In the sequel we present some examples of $I I$-minimal hypersurfaces in spheres.

Example 1. The standard embedding $\mathbb{S}^{n-1}(r) \hookrightarrow \mathbb{S}^{n}(1)$ is $I I$ minimal if and only if $r=\frac{1}{\sqrt{2}}$.

Proof: Let $\left(x^{0}, x^{1}, \ldots, x^{n}\right)$ be global coordinates in $\mathbb{E}^{n+1}$ and denote by $p$ either the point or the position vector in $\mathbb{E}^{n+1}$. Without loss of the generality one can think the sphere $\mathbb{S}^{n-1}(r)$ as obtained by cutting the unit sphere $\mathbb{S}^{n}$ by the hyperplane $x^{0}=\sqrt{1-r^{2}}$. Thus, an arbitrary vector field tangent to $\mathbb{S}^{n-1}(r)$ can be expressed as $X=\left(0, X^{1}, \ldots, X^{n}\right)$, where $X^{i}$ are smooth functions depending on $x^{1}, \ldots, x^{n}$ such that $\sum_{i=1}^{n} X^{i} x^{i}=0$. In order to compute the second mean curvature, one can express first the unit normal of the embedding as $\nu= \pm\left(-r, \frac{\sqrt{1-r^{2}}}{r}\left(x^{1}, \ldots, x^{n}\right)\right)$. Fixing an orientation we choose e.g. the "plus" sign. The second fundamental form is given by $I I(X, Y)=-\frac{\sqrt{1-r^{2}}}{r}\langle X, Y\rangle$ for all $X, Y \in T\left(\mathbb{S}^{n-1}(r)\right)$, where $\langle$,$\rangle denotes the usual Euclidean scalar product.$ Note that $I I$ is negatively defined. We are able now to obtain the second mean curvature. After straightforward computations in (2) one gets

$$
H_{I I}=\frac{1}{2}\left(\frac{r}{\sqrt{1-r^{2}}}-\frac{\sqrt{1-r^{2}}}{r}\right) .
$$

Under the assumption of $I I$-minimality, $H_{I I}=0$ is equivalent with $r=\frac{1}{\sqrt{2}}$. Hence the conclusion.

More generally we have 
Example 2. The embedding $\mathbb{S}^{n-1}(r) \hookrightarrow \mathbb{S}^{n}(R)$ is $I I$-minimal if and only if $r=$ $\frac{R}{\sqrt{R^{2}+1}}$.

Proof: Similar computations as in previous example.

Remark 1. The following chain of embeddings

$$
\mathbb{S}^{1}\left(\frac{1}{\sqrt{n}}\right) \hookrightarrow \mathbb{S}^{2}\left(\frac{1}{\sqrt{n-1}}\right) \hookrightarrow \cdots \hookrightarrow \mathbb{S}^{n-1}\left(\frac{1}{\sqrt{2}}\right) \hookrightarrow \mathbb{S}^{n}(1)
$$

is such that each embedding $\mathbb{S}^{k}\left(\frac{1}{\sqrt{n-k+1}}\right) \hookrightarrow \mathbb{S}^{k+1}\left(\frac{1}{\sqrt{n-k}}\right)$ is $I I$-minimal for any $k \in\{1, \ldots, n-1\}$.

Let us give another example of a $I I$-minimal surface in the unit sphere $\mathbb{S}^{3}$.

Example 3. Let us consider the following parametrization

$$
r: M \longrightarrow \mathbb{S}^{3}(1), r(s, t)=(\cos s \cos t, \sin s \cos t, \cos s \sin t, \sin s \sin t) .
$$

Then second mean curvature of the surface $M$ vanishes identically.

Proof: The proof of this statement is straightforward.

The next example ends the series of $I I$-minimal surfaces in spheres presented in this paper.

Example 4. Let $M=\mathbb{S}^{m_{1}}\left(r_{1}\right) \times \mathbb{S}^{m_{2}}\left(r_{2}\right) \hookrightarrow \mathbb{S}^{m+1}(r)=\widetilde{M}$ be the usual embedding with $r_{1}^{2}+r_{2}^{2}=r^{2}$ and $m_{1}+m_{2}=m$. Then, $M$ is $I I$-minimal in $\widetilde{M}$ if and only if $r_{1}=r \sqrt{\frac{m_{1}+m_{2} r^{2}}{m\left(r^{2}+1\right)}}$ and $r_{2}=r \sqrt{\frac{m_{2}+m_{1} r^{2}}{m\left(r^{2}+1\right)}}$.

Proof: Consider $\left(x^{0}, x^{1}, \ldots, x^{m_{1}}\right)$ and $\left(y^{0}, y^{1}, \ldots, y^{m_{2}}\right)$ global coordinates on $\mathbb{R}^{m_{1}+1}$, respectively on $\mathbb{R}^{m_{2}+1}$. The unit normal is $\nu=\frac{1}{r}\left(-\frac{r_{2}}{r_{1}} x, \frac{r_{1}}{r_{2}} y\right)$ where $x=\left(x^{0}, x^{1}, \ldots, x^{m_{1}}\right)$ and $y=\left(y^{0}, y^{1}, \ldots, y^{m_{2}}\right)$. Hence the Weingarten operator can be expressed as

$$
A=\left(\begin{array}{cc}
\frac{r_{2}}{r r_{1}} I_{m_{1}} & O \\
O & -\frac{r_{1}}{r r_{2}} I_{m_{2}}
\end{array}\right)
$$

whose determinant is constant. By $I_{k}$ we denoted the identity $k \times k$ matrix. After straightforward computations we get from (2) that the second mean curvature is given by

$$
H_{I I}=\frac{1}{2 r r_{1} r_{2}}\left(m_{1} r_{2}^{2}-m_{2} r_{1}^{2}-m_{1} r^{2} r_{1}^{2}+m_{2} r^{2} r_{2}^{2}\right) .
$$

Hence the conclusion. 
Remark 2. In particular, if $m=2$ and $r=1$ we get that the Clifford torus

$$
\mathbb{S}^{1}\left(\frac{1}{\sqrt{2}}\right) \times \mathbb{S}^{1}\left(\frac{1}{\sqrt{2}}\right) \hookrightarrow \mathbb{S}^{3}(1)
$$

is $I I$-minimal.

Proof: See for example [7].

Having in mind these results, there is another interesting property involving the curvatures of a surface that we can study, the Weingarten property. If $A, B$ are two different type curvatures of a (non-developable) surface, and if there is a nontrivial functional relation between $A$ and $B$, then the surface is called an $\{A, B\}-$ generalized Weingarten surface. See for details [4].

Remark 3. The Clifford torus is a $\left\{H, H_{I I}\right\}$-generalized Weingarten surface.

Proof: Easy computations yield the following relation: $H_{I I}=2 H$, namely, the mean curvature corresponding to the first fundamental form $H$ and the mean curvature corresponding to the second fundamental form $H_{I I}$ are proportional.

Seeing all these nice examples for spheres, we wonder what happens when the ambient is the hyperbolic space. At this point, if we consider similar problems, e.g. II-minimality, in the hyperbolic spaces, we get the following non-existence result:

Proposition 1. There is no $r>0$ such that $\mathbb{H}^{n-1}(-r) \hookrightarrow \mathbb{H}^{n}(-1)$ is $I I$-minimal. Here $\mathbb{H}_{R}^{k}:=\mathbb{H}^{k}(-R)=\left\{x \in \mathbb{R}_{1}^{k+1} ;\langle x, x\rangle_{1}=-R^{2}, x_{0}>0\right\}(R>0)$ where $\langle,\rangle_{1}$ denotes the usual Lorentzian scalar product with signature $(-+\cdots+)$.

Proof: After similar computations as in the previous examples we find the expression of the second mean curvature, but under the restriction of $I I$-minimality we reach a contradiction!

Having in mind all these examples in spheres and in hyperbolic spaces, let us recall now another interesting property for surfaces and hypersurfaces, the biharmonicity. As the aim of our article does not consist in the study of biharmonicity, the reader is invited to check [1] for more details on the subject.

In the end of this section we wold like to bring into attention some classical results concerning the biharmonicity of the surfaces and hypersurfaces above studied form the $I I$-minimality point of view. Concerning the spheres, it is known that the proper biharmonic surfaces in $\mathbb{S}^{3}$ are also $I I$-minimal surfaces. Moreover, the hyperspheres $\mathbb{S}^{m}\left(\frac{1}{\sqrt{2}}\right)$ and the generalized Clifford tori $\mathbb{S}^{m_{1}}\left(\frac{1}{\sqrt{2}}\right) \times \mathbb{S}^{m_{2}}\left(\frac{1}{\sqrt{2}}\right)$, $m_{1} \neq m_{2}$ are the only known examples of proper biharmonic hypersurfaces in $\mathbb{S}^{m+1}$. 
If the problem is considered in hyperbolic spaces, only few results are obtained. For example [1], there exist no proper biharmonic hypersurfaces in $\mathbb{H}^{4}$.

\section{II-minimal translation surfaces}

In this section we analyze II-minimal translation surfaces with Riemannian second fundamental form, namely we study under which conditions the second mean curvature vanishes, i.e. $H_{I I}=0$. Having in mind the usual technique for computing the second mean curvature by using the normal variation of the area functional one gets for surfaces in $\mathbb{E}^{3}$

$$
H_{I I}=H+\frac{1}{4} \Delta_{I I} \log (K)
$$

where $K$ and $H$ denote the usual Gaussian, respectively mean curvatures of our surface and $\Delta_{I I}$ is the Laplacian for functions computed with respect to the second fundamental form as metric. $H_{I I}$ can be equivalently expressed as

$$
H_{I I}=H+\frac{1}{2 \sqrt{\operatorname{det} I I}} \sum_{i, j} \frac{\partial}{\partial u^{i}}\left(\sqrt{\operatorname{det} I I} h^{i j} \frac{\partial}{\partial u^{j}}(\ln \sqrt{K})\right) .
$$

Here $I I$ denotes the second fundamental form, $\left(h_{i j}\right)$ is the associated matrix with its inverse $\left(h^{i j}\right)$, the indices $i, j$ belong to $\{1,2\}$ and the parameters $u^{1}, u^{2}$ are $x$, respectively $y$ from the parametrization (1). Moreover, $I I$ becomes a metric on the surface if it is positive definite (or, more generally, if it is non-degenerated). Sometimes, the second mean curvature is taken with the opposite sign (see [12]).

Accordingly to parametrization (1) of a translation surface and denoting $r$ the corresponding immersion in the Euclidean 3-space endowed with the Euclidean scalar product $\langle$,$\rangle , namely r: M \rightarrow \mathbb{E}^{3},(x, y) \mapsto r(x, y)=(x, y, f(x)+g(y))$ one easily computes its first fundamental form

$$
I=E \mathrm{~d} x^{2}+2 F \mathrm{~d} x \mathrm{~d} y+G \mathrm{~d} y^{2}
$$

where $E, F, G$ - the coefficients of $I$ - are given by $E=\left\langle r_{x}, r_{x}\right\rangle, F=\left\langle r_{x}, r_{y}\right\rangle$, $G=\left\langle r_{y}, r_{y}\right\rangle$ and the second fundamental form

$$
I I=L \mathrm{~d} x^{2}+2 M \mathrm{~d} x \mathrm{~d} y+N \mathrm{~d} y^{2}
$$

with the coefficients given by $L=\frac{\left(r_{x}, r_{y}, r_{x x}\right)}{\sqrt{E G-F^{2}}}, M=\frac{\left(r_{u} x, r_{y}, r_{x y}\right)}{\sqrt{E G-F^{2}}}$ and $N=\frac{\left(r_{x}, r_{y}, r_{y y}\right)}{\sqrt{E G-F^{2}}}$. Denoting $f^{\prime}=\alpha$ and $g^{\prime}=\beta$, we get

$$
\begin{gathered}
I=\left(1+\alpha(x)^{2}\right) \mathrm{d} x^{2}+2 \alpha(x) \beta(y) \mathrm{d} x \mathrm{~d} y+\left(1+\beta(y)^{2}\right) \mathrm{d} y^{2} \\
I I=\frac{1}{\sqrt{\Delta}}\left(\alpha^{\prime}(x) \mathrm{d} x^{2}+\beta^{\prime}(y) \mathrm{d} y^{2}\right)
\end{gathered}
$$

where $\Delta=1+\alpha(x)^{2}+\beta(y)^{2}$. 
The inverse matrix $\left(h^{i j}\right)$ of the second fundamental form of a translation surface has the following expression

$$
\left(h^{i j}\right)_{i, j}=\left(\begin{array}{cc}
\frac{\sqrt{1+\alpha^{2}+\beta^{2}}}{\alpha^{\prime}} & 0 \\
0 & \frac{\sqrt{1+\alpha^{2}+\beta^{2}}}{\beta^{\prime}}
\end{array}\right) .
$$

The curvatures corresponding to the first fundamental form, the Gaussian curvature $K=\frac{L N-M^{2}}{E G-F^{2}}$ and and the mean curvature $H=\frac{E N-2 F M+G L}{E G-F^{2}}$ become in this case

and

$$
K=\frac{\alpha^{\prime}(x) \beta^{\prime}(y)}{\Delta^{2}}
$$

$$
H=\frac{\left(1+\beta^{2}(y)\right) \alpha^{\prime}(x)+\left(1+\alpha^{2}(x)\right) \beta^{\prime}(y)}{2 \Delta^{3 / 2}} .
$$

After straightforward computations, the sum in (5) has the following expression

$$
\begin{aligned}
\sum_{i, j}= & \frac{1}{4 \Delta^{2}} \sqrt{\frac{\beta^{\prime}}{\alpha^{\prime}}}\left(\frac{2 \alpha^{\prime} \alpha^{\prime \prime \prime}-3 \alpha^{\prime \prime 2}}{\alpha^{\prime 2}} \Delta^{2}+\left(-4 \alpha \alpha^{\prime \prime}-8 \alpha^{\prime 2}\right) \Delta+16 \alpha^{2} \alpha^{2}\right)+ \\
& +\frac{1}{4 \Delta^{2}} \sqrt{\frac{\alpha^{\prime}}{\beta^{\prime}}}\left(\frac{2 \beta^{\prime} \beta^{\prime \prime \prime}-3 \beta^{\prime \prime 2}}{\beta^{\prime 2}} \Delta^{2}+\left(-4 \beta \beta^{\prime \prime}-8 \beta^{\prime 2}\right) \Delta+16 \beta^{2} \beta^{2}\right) .
\end{aligned}
$$

Notice that $\alpha^{\prime} \beta^{\prime}>0$ since the second fundamental form is positive definite, so the square roots are defined.

We are interested to find $I I$-minimal translation surfaces in the Euclidean 3-space. Having now all the necessary tools, the condition $H_{I I}=0$ for a translation surface is equivalent to

$$
\begin{gathered}
\frac{2 \alpha^{\prime} \alpha^{\prime \prime \prime}-3 \alpha^{\prime \prime 2}}{2 \alpha^{\prime 3}}+\frac{2 \beta^{\prime} \beta^{\prime \prime \prime}-3 \beta^{\prime \prime 2}}{2 \beta^{\prime 3}}-\frac{2}{\Delta}\left(\frac{\alpha^{\prime 2}+\alpha \alpha^{\prime \prime}}{\alpha^{\prime}}+\frac{\beta^{\prime 2}+\beta \beta^{\prime \prime}}{\beta^{\prime}}\right)+ \\
+\frac{6}{\Delta^{2}}\left(\alpha^{2} \alpha^{\prime}+\beta^{2} \beta^{\prime}\right)=0
\end{gathered}
$$

The first two terms in (6) are functions only of $x$ respectively of $y$, hence we take the derivatives in the previous equation successively w.r.t. $x$ and $y$.

Denoting by

$$
\phi(x)=\frac{\alpha^{\prime 2}+\alpha \alpha^{\prime \prime}}{\alpha^{\prime}}, \psi(y)=\frac{\beta^{\prime 2}+\beta \beta^{\prime \prime}}{\beta^{\prime}}, p(x)=\alpha^{2} \alpha^{\prime} \text { and } q(y)=\beta^{2} \beta^{\prime}
$$

we get

$$
\frac{\partial}{\partial y} \frac{\partial}{\partial x}\left(-\frac{2}{\Delta}(\phi+\psi)+\frac{6}{\Delta^{2}}(p+q)\right)=0 .
$$

After straightforward computations and multiplying with $\frac{\Delta^{3}}{8 \alpha \alpha^{\prime} \beta \beta^{\prime}}$ it follows

$$
(F+G) \Delta^{2}-2(P+Q) \Delta+18(p+q)=0
$$


where $F(x)=\frac{\phi^{\prime}}{2 \alpha \alpha^{\prime}}, G(y)=\frac{\psi^{\prime}}{2 \beta \beta^{\prime}}, P(x)=\phi+\frac{3 p^{\prime}}{2 \alpha \alpha^{\prime}}$ and $Q(y)=\psi+\frac{3 q^{\prime}}{2 \beta \beta^{\prime}}$.

Repeating similar operations, namely taking the two partial derivatives and dividing by $4 \alpha \alpha^{\prime} \beta \beta^{\prime}$ one gets

$$
(A+B) \Delta+a+b=0
$$

where $A(x)=\frac{F^{\prime}}{2 \alpha \alpha^{\prime}}, B(y)=\frac{G^{\prime}}{2 \beta \beta^{\prime}}, a(x)=F-\frac{P^{\prime}}{2 \alpha \alpha^{\prime}}$ and $b(y)=G-\frac{Q^{\prime}}{2 \beta \beta^{\prime}}$.

Finally, using the same technique, we should have

$$
\frac{A^{\prime}}{2 \alpha \alpha^{\prime}}=c \quad \frac{B^{\prime}}{2 \beta \beta^{\prime}}=-c, \quad c \in \mathbb{R} .
$$

Solving the above equations we obtain $A(x)=c \alpha^{2}+d_{1}$ and $B(y)=-c \beta^{2}+d_{2}$. Replacing these expressions in the previous ODEs we find that

$$
\begin{aligned}
& F(x)=\frac{c}{2} \alpha^{4}+d_{1} \alpha^{2}+\mu_{1} \\
& G(y)=-\frac{c}{2} \beta^{4}+d_{2} \beta^{2}+\mu_{2} \\
& \phi(x)=\frac{c}{6} \alpha^{6}+\frac{d_{1}}{2} \alpha^{4}+\mu_{1} \alpha^{2}+\tau_{1} \\
& \psi(y)=-\frac{c}{6} \beta^{6}+\frac{d_{2}}{2} \beta^{4}+\mu_{2} \beta^{2}+\tau_{2} \\
& \alpha^{\prime}(x)=\frac{c}{42} \alpha^{6}+\frac{d_{1}}{10} \alpha^{4}+\frac{\mu_{1}}{3} \alpha^{2}+\tau_{1}+\frac{\rho_{1}}{\alpha} \\
& \beta^{\prime}(y)=-\frac{c}{42} \beta^{6}+\frac{d_{2}}{10} \beta^{4}+\frac{\mu_{2}}{3} \beta^{2}+\tau_{2}+\frac{\rho_{2}}{\beta} \\
& p(x)=\frac{c}{42} \alpha^{8}+\frac{d_{1}}{10} \alpha^{6}+\frac{\mu_{1}}{3} \alpha^{4}+\tau_{1} \alpha^{2}+\rho_{1} \alpha \\
& q(y)=-\frac{c}{42} \beta^{8}+\frac{d_{2}}{10} \beta^{6}+\frac{\mu_{2}}{3} \beta^{4}+\tau_{2} \beta^{2}+\rho_{2} \beta \\
& P(x)=\frac{19 c}{42} \alpha^{6}+\frac{7 d_{1}}{5} \alpha^{4}+3 \mu_{1} \alpha^{2}+4 \tau_{1}+\frac{3 \rho_{1}}{2 \alpha} \\
& Q(y)=-\frac{19 c}{42} \beta^{6}+\frac{7 d_{2}}{5} \beta^{4}+3 \mu_{2} \beta^{2}+4 \tau_{2}+\frac{3 \rho_{2}}{2 \beta} \\
& a(x)=-\frac{6 c}{7} \alpha^{4}-\frac{9 d_{1}}{5} \alpha^{2}-2 \mu_{1}+\frac{3 \rho_{1}}{4 \alpha^{3}} \\
& b(y)=\frac{6 c}{7} \beta^{4}-\frac{9 d_{2}}{5} \beta^{2}-2 \mu_{2}+\frac{3 \rho_{2}}{4 \beta^{3}}
\end{aligned}
$$

where $d_{1}, d_{2}, \mu_{1}, \mu_{2}, \tau_{1}, \tau_{2}, \rho_{1}, \rho_{2} \in \mathbb{R}$. In order to determine all these integration constants, we substitute the corresponding expressions in (8), obtaining a sum of polynomials in $\alpha$ and $\beta$ equals to 0 . This means that there exists $\xi \in \mathbb{R}$ such that

$$
\begin{gathered}
\frac{c}{7} \alpha^{4}+\left(c-\frac{4}{5} d_{1}+d_{2}\right) \alpha^{2}+\frac{3 \rho_{1}}{4 \alpha^{3}}+d_{1}-2 \mu_{1}-\xi=0 \\
-\frac{c}{7} \beta^{4}+\left(-c+d_{1}-\frac{4}{5} d_{2}\right) \beta^{2}+\frac{3 \rho_{2}}{4 \beta^{3}}+d_{2}-2 \mu_{2}+\xi=0 .
\end{gathered}
$$


At this point, by the same argument as in previous section, all the coefficients in the above (algebraic) expressions must be zero and consequently we get $c=0$, $d_{1}=d_{2}=0, \rho_{1}=\rho_{2}=0, \mu_{1}=-\frac{\xi}{2}$ and $\mu_{2}=\frac{\xi}{2}$. Thus, the previous expressions can be expressed in a simpler form

$$
\begin{array}{ll}
F(x)=-\frac{\xi}{2} & G(y)=\frac{\xi}{2} \\
\phi(x)=-\frac{\xi}{2} \alpha^{2}+\tau_{1} & \psi(y)=\frac{\xi}{2} \beta^{2}+\tau_{2} \\
\alpha^{\prime}(x)=-\frac{\xi}{6} \alpha^{2}+\tau_{1} & \beta^{\prime}(y)=\frac{\xi}{6} \beta^{2}+\tau_{2} \\
p(x)=-\frac{\xi}{6} \alpha^{4}+\tau_{1} \alpha^{2} & q(y)=\frac{\xi}{6} \beta^{4}+\tau_{2} \beta^{2} \\
P(x)=-\frac{3 \xi}{2} \alpha^{2}+4 \tau_{1} & Q(y)=\frac{3 \xi}{2} \beta^{2}+4 \tau_{2} \\
a(x)=\xi & b(y)=-\xi .
\end{array}
$$

Let us take a look in (7). By the same reasoning as above we deduce

$$
\begin{aligned}
& \left(3 \xi+10 \tau_{1}-8 \tau_{2}\right) \alpha^{2}-8 \tau_{1}=\eta \\
& \left(-3 \xi-8 \tau_{1}+10 \tau_{2}\right) \beta^{2}-8 \tau_{2}=-\eta
\end{aligned}
$$

for an arbitrary $\eta \in \mathbb{R}$. Moreover, the integration constants should be $\tau_{1}=-\frac{\eta}{8}$, $\tau_{2}=\frac{\eta}{8}, \xi=\frac{3 \eta}{4}$ and we can conclude that $\alpha^{\prime}=-\frac{\eta}{8}\left(\alpha^{2}+1\right)$ and $\beta^{\prime}=\frac{\eta}{8}\left(\beta^{2}+\right.$ $1)$. Finally $\alpha$ and $\beta$ must satisfy also the condition (6). After straightforward computations it follows that $\eta=\xi=0$.

The conclusion is $\alpha^{\prime}=\beta^{\prime}=0$, which cannot occur since if this happened the second fundamental form would vanish identically. Hence the second mean curvature is not defined and we end this section with the following non-existence theorem.

Theorem 1. There are no II-minimal translation surfaces in Euclidean 3-space.

\section{Acknowledgements}

The first author was supported by grant PN-II ID 398/2007-2010, CNCSIS, Romania. The second author was partially supported by grant PN-II ID 457/2009-2011, CNCSIS, Romania.

\section{References}

[1] Balmuş A., Biharmonic Maps and Submanifolds, PhD. Thesis, Universtà degli Studi di Cagliari, Italy, 2007.

[2] Cartan E., Les surfaces qui Admettent une Seconde Forme Fondamentale Donnée, Bull. Sci. Math. 67 (1943) 2 8-32.

[3] Darboux G., Leçons sur la théorie générale des surfaces, Gauthiers-Villars, Paris 1915. 
[4] Dillen F. and Sodsiri W., Ruled Surfaces of Weingarten Type in Minkowski 3-space, J. of Geometry, 83 (2005) 1-2, 10-21.

[5] Erard P., Über die zweite Fundamentalform von Flächen im Raum, Abhandlung zur Erlangung der Würde eines Doktors der Mathematik der Eidgenössischen Technischen Hochschule Zürich. Diss. No. 4234, ETH Zürich, 1968.

[6] Glässner E., Über die Minimalflächen der Zweiten Fundamentalform, Monatsh. Math. 78 (1974) 193-214.

[7] Haesen S., Verpoort S. and Verstraelen L., The Mean Curvature of the Second Fundamental Form, Houston J. of Math. 34 (2008) 3 703-719.

[8] Manhart F., Die II-Minimalregelflächen, Anzeiger der Österreichische Akademie der Wissenschaften. Mathematisch-Naturwissenschaftliche Klasse, 119 (1982) 157-160.

[9] Manhart F., Die Affinminimalrückungsflächen, Arch. Math. 44 (1985) 547-556.

[10] Munteanu M. and Nistor A., On the Geometry of the Second Fundamental Form of Translation Surfaces in $\mathbb{E}^{3}$, to appear in Houston J. Math. (2010), arXiv:0812.3166v1 [math.DG].

[11] Selig J., Geometric Fundamentals of Robotics, Second Edition, Springer, 2005.

[12] Verpoort S., The Geometry of the Second Fundamental Form: Curvature Properties and Variational Aspects, PhD. Thesis, Katholieke Universiteit Leuven, Belgium, 2008.

[13] Weingarten J., Über die Deformationen einer biegsamen unausdehnbaren Fläche, Journal für die Reine und Angewändte Mathematik (Crelle's Journal) 100 (1887), 296-310. 